\title{
DAMPING OF COHERENT OSCILLATIONS
}

\author{
L. Vos
}

CERN, CH-1211 Geneva 23, Switzerland

Damping of coherent oscillations by feedback is straightforward in principle. It has been a vital ingredient for the safe operation of accelerators since a long time [1,2]. The increasing dimensions and beam intensities of the new generation of hadron colliders impose unprecedented demands on the performance of future systems. The arguments leading to the specification of a transverse feedback system for the CERN SPS in its role as LHC injector and the LHC collider itself are developed to illustrate this. The preservation of the transverse emittance is the guiding principle during this exercise keeping in mind the hostile environment which comprises: transverse impedance bent on developing coupled bunch instabilities, injection errors, unwanted transverse excitation, unavoidable tune spreads and noise in the damping loop.

\section{Introduction}

The traditional task of a transverse feedback system is to stabilise the beam against the low frequency instability mechanism provoked by the resistive wall impedance. This aspect lays the foundation of the specification of such systems and the SPS and the LHC are no exception to this. Their definition is sharpened when injection oscillations and the rate at which they need to be damped are included in the picture. This in fact opens the chapter of emittance conservation which is vital for hadron colliders, hence imperative for their injectors. The requirements of their transverse feedback are further focused by considering the emittance conservation issue during the more quiet periods of the accelerator cycle, i.e. injection coast between injections and acceleration. A last but not least consideration concerns the same emittance conservation issue during the long collider coasts.

A simplified diagram of a transverse feedback system is shown in Figure 1.

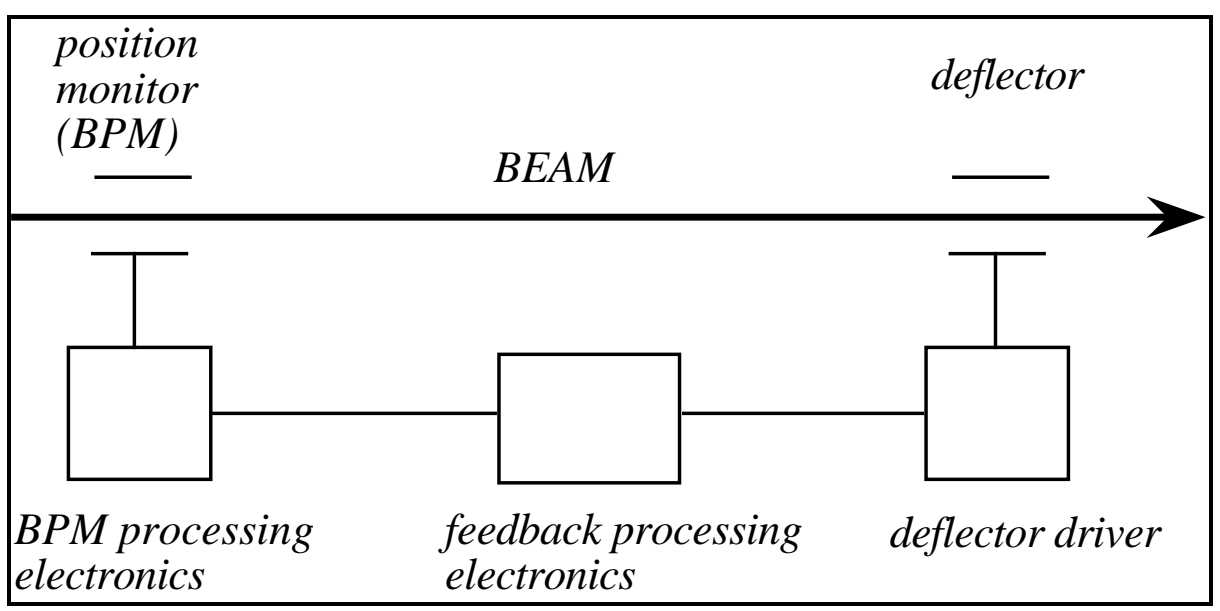

Figure 1 : Transverse feedback system 
The position monitor picks up signals from a portion of the beam. These signals are treated first by the monitor electronics and are then passed on to the feedback processing and power electronics which drives the deflector. The delays must be accurate enough so that measurement and deflection are related to exactly the same beam portion within the time resolution of the system. Since the monitor is sensitive to position and the kicker acts on angle it is necessary to ensure a correct odd multiple of N4 betatron wavelengths between the two devices. The time delay between measurement and correction is about 1 machine turn for reasons of layout in these large machines. The feedback electronics ensures the correct operation of the system. Its task is complicated $[3,4,5,6]$ and therefore it is to be expected that digital signal processing will be even more important than it is today.

The aim of this paper is not so much to design the hardware of transverse feedback systems but rather to answer basic queries that will be raised inevitably when hardware and technology choices have to be made :

- what is the bandwidth and what is the best operating frequency?

- what is the deflection strength and is it needed over the full bandwidth?

- what is the position resolution?

- what is the time resolution?

- what is the gain?

- what is the dynamic range?

The following exercise on transverse feedback concerns the LHC and the SPS as its injector. They handle similar beams justifying the comparison between the two. Feedback is needed in the two transverse planes. The vertical plane is the most demanding one and to keep the argumentation as clear as possible, only that one will be studied. Relevant machine and beam parameters are shown in Table 1.

\begin{tabular}{|c|c|c|c|c|c|}
\hline & \multicolumn{2}{|c|}{ SPS } & \multicolumn{2}{|c|}{$\mathrm{LHC}$} \\
\hline \multirow{5}{*}{$\begin{array}{l}\text { momentum E/ec } \\
\text { machine radius } R \\
\text { pipe radius } b \\
\text { revolution time/frequency } \\
T / f_{\text {rev }}\end{array}$} & \multirow{4}{*}{$\begin{array}{l}\mathrm{GeV} / \mathrm{c} \\
\mathrm{m} \\
\mathrm{mm} \\
\mu \mathrm{s} / \mathrm{kHz}\end{array}$} & 26 & 450 & 450 & 7000 \\
\hline & & \multicolumn{2}{|c|}{1100} & \multicolumn{2}{|c|}{4242} \\
\hline & & \multirow{2}{*}{\multicolumn{2}{|c|}{$\begin{array}{c}25 \\
23 / 43.3\end{array}$}} & \multirow{3}{*}{\multicolumn{2}{|c|}{$\begin{array}{c}18 \\
89 / 11.2\end{array}$}} \\
\hline & & & & & \\
\hline & & \multirow{2}{*}{\multicolumn{2}{|c|}{26.6}} & & \\
\hline$Q$ & & & & \multicolumn{2}{|c|}{63.3} \\
\hline $\mathrm{Nb}$ of bunches & & \multicolumn{2}{|c|}{243} & \multicolumn{2}{|c|}{2835} \\
\hline particles/bunch $n$ & $10^{11}$ & \multicolumn{2}{|c|}{1.7} & \multicolumn{2}{|c|}{1.7} \\
\hline total intensity $i_{\text {tot }}$ & $A$ & \multicolumn{2}{|c|}{0.29} & \multicolumn{2}{|c|}{0.87} \\
\hline bunch spacing & & \multirow{2}{*}{\multicolumn{2}{|c|}{$\begin{array}{l}25 \\
40\end{array}$}} & \multirow{2}{*}{\multicolumn{2}{|c|}{$\begin{array}{l}25 \\
40\end{array}$}} \\
\hline bunch frequency $f_{b}$ & $M H z$ & & & & \\
\hline bunch length $\sigma_{t} / \sigma_{s}$ & $\mathrm{~ns} / \mathrm{mm}$ & $1 / 300$ & $0.434 / 130$ & $0.434 / 130$ & $0.257 / 77$ \\
\hline peak intensity $\hat{i}$ & & 10.9 & 25 & 25 & 42 \\
\hline transverse emittance $\varepsilon$ & $\mu \mathrm{radm}$ & 3 & 3.5 & 3.5 & 3.75 \\
\hline average radius $\sigma$ & $\mathrm{mm}$ & 2.3 & 0.6 & 0.7 & 0.2 \\
\hline
\end{tabular}

Table 1: General beam and machine parameters of SPS and LHC 
It may be important to point out that the chosen beam and bunch intensities are those that are related to the ultimate LHC beam. All beam dimensions are r.m.s..

\section{Bandwidth and frequency}

The beam samples the machine impedance at the bunch repetition frequency $f_{b}$. The maximum frequency that can be observed is limited to $f_{b} / 2$ (Nyquist). This then fixes the maximum required bandwidth of a feedback system that is asked to stabilise transverse rigid bunch motion. Higher frequencies than $f_{b} / 2$ will be down-sampled to the so-called baseband. The main instability mechanism that a feedback has to handle is the low frequency narrow-band resistive wall impedance which falls off with frequency. Since the r.m.s. length of proton bunches is generally much larger than the vacuum chamber radius it is rare that other narrow band resonances occur at frequencies between the bunch repetition frequency $f b$ and the bunch r.m.s. spectral frequency $\langle f\rangle=1 / 2 \pi \sigma_{\mathrm{t}}$. Typical values for $\langle f\rangle$ are $160 \mathrm{MHz}$ in the SPS and 370 $\mathrm{MHz}$ in the LHC. The system delay (position monitor to transverse kick ) has to be constant within $1 / f_{b}$.

It is a good principle to correct an error at its origin. In this case the origin of the perturbations is the low frequency resistive wall instability which should ideally be observed and eliminated in the base-band. However, it may be necessary for technological reasons to depart from that principle [28]. If the bunches were infinitely short and if no other perturbations were present, then operation at any higher frequency mode would be acceptable and equally effective. However, bunches are not infinitely short and working at a frequency band different from the base-band will reduce the quality of the operation.

For signal observation it is quite acceptable to depart from the baseband if the beams are bunched at all times. The transfer function of the monitor and its processing can be known precisely in the required band width of $\pm f_{b} / 2$. All the modes below $\langle f\rangle$ carry the same information at comparable signal level.

The correction signal imparted to the beam by the deflector has to obey very strict rules. Among others, it has to be well matched to the observed signal. This condition is formally fulfilled only when the deflector is working in base-band. The convolution between the correction and the bunch spectrum deviates more and more from the ideal situation for higher modes. The phase shift between the base band correction and the higher mode correction will cause blow-up of the bunch edges. That feature may be appreciated from Figure 2. The time profile of a correction at baseband(ideal correction) is compared with the time profiles of corrections done at half and at full r.m.s. bunch spectral frequency $\langle f\rangle$. 


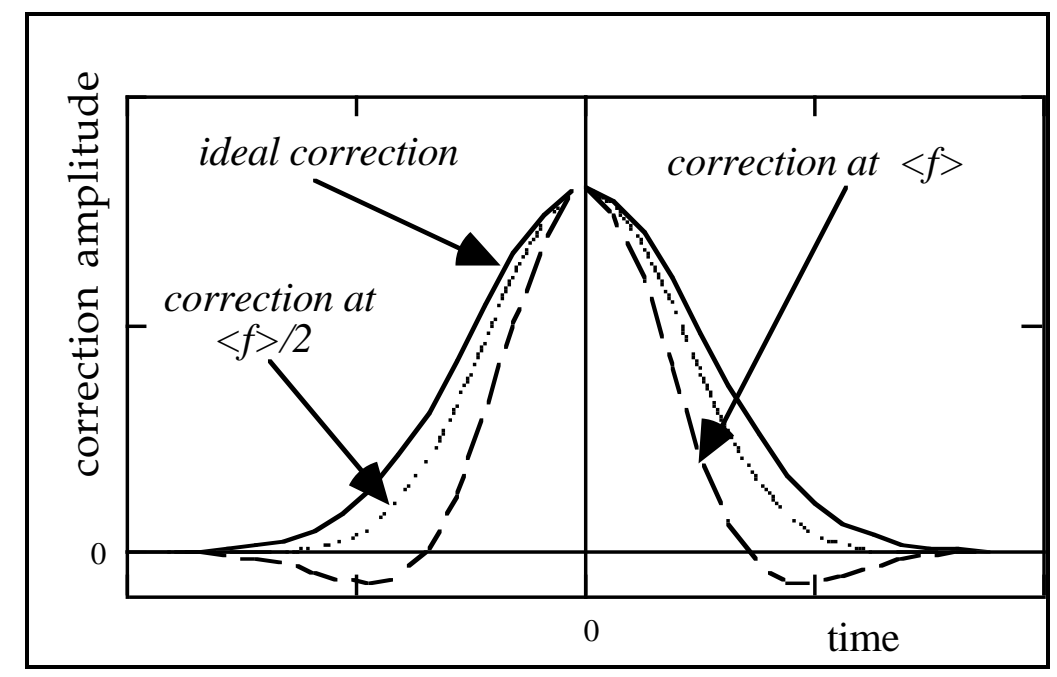

Figure 2 : Comparison between base-band and high frequency corrections

It looks a safe criterion to keep the deflector frequency below $\langle f\rangle / 2$ (80 $\mathrm{MHz}$ in the SPS and $180 \mathrm{MHz}$ in the LHC). Moreover, it is worth to mention that synchronisation errors at higher modes are much more critical than for base-band operation.

\section{Resistive wall impedance and feedback}

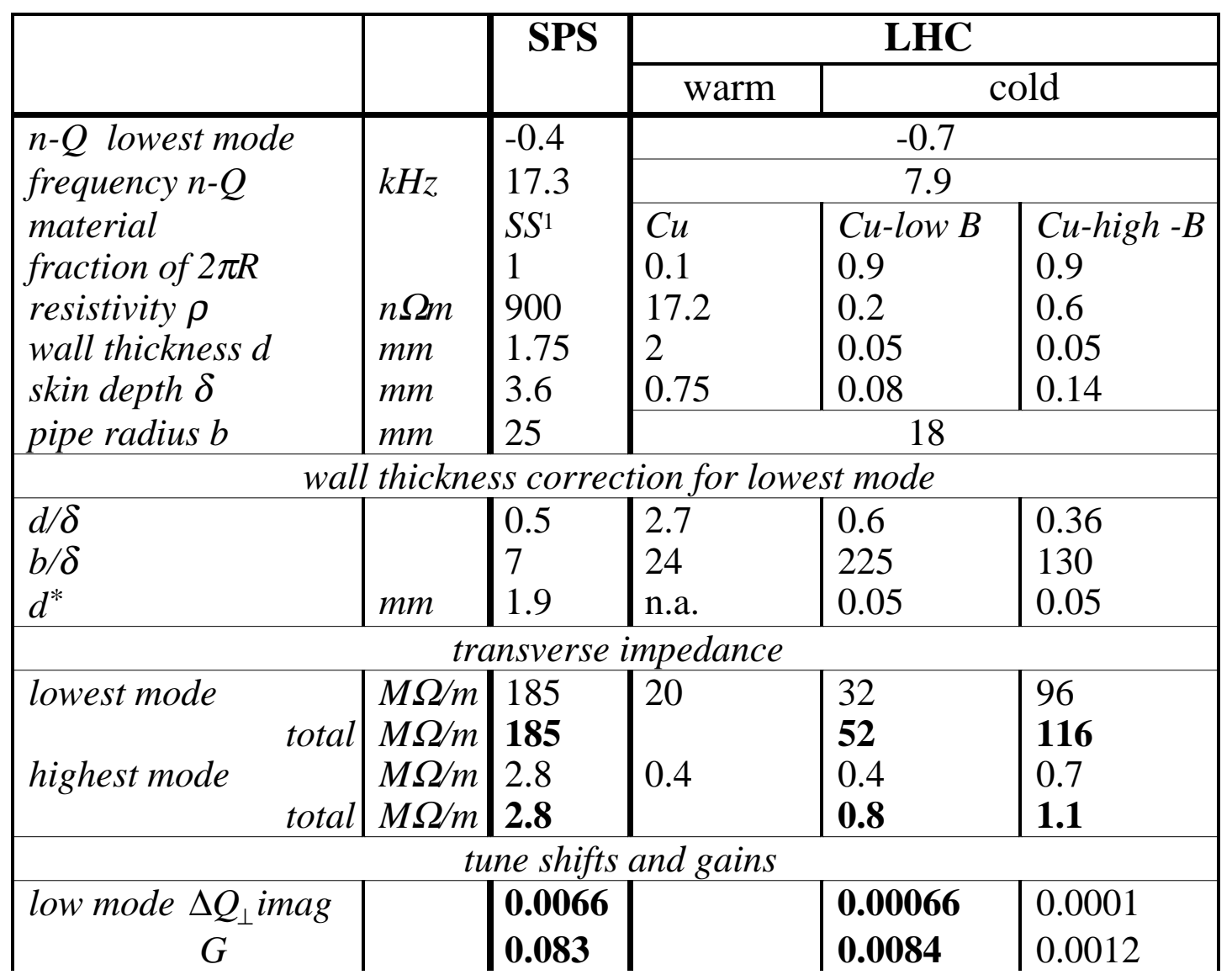

${ }^{1} S S=$ stainless steel 


\begin{tabular}{|c|c|c|c|}
\hline $\begin{array}{c}\text { high mode } \\
G\end{array} Q_{\perp}$ imag & $\begin{array}{l}0.0004 \\
0.005\end{array}$ & $\begin{array}{l}0.00001 \\
0.00014\end{array}$ & $\begin{array}{l}0.000001 \\
0.000011\end{array}$ \\
\hline
\end{tabular}

Table 2 : Resistive wall impedance in SPS and LHC

A resistive impedance causes in general an imaginary tune shift and a real risetime. The relation between tune shift $\Delta Q_{\perp}$ and transverse impedance $Z_{\perp}$ is :

$$
\Delta Q_{\perp}=\frac{1}{4 \pi} \frac{R / Q}{E / e} Z_{\perp} i
$$

where $i$ is the beam intensity that participates in the instability. The instability rise time $\tau$ is :

$$
\frac{\tau}{T}=\frac{1}{2 \pi \Delta Q_{\perp}} .
$$

A feedback that compensates an imaginary tune shift of $\Delta Q_{\perp}$ has a gain $G$ of :

$$
G=\frac{1}{4 \pi \Delta Q_{\perp}} .
$$

$G$ is the ratio of the deflection at the output to the position at the input, both expressed in normalised units.

The following standard relation between longitudinal $Z_{1}$ and transverse impedance will be used where necessary :

$$
Z_{\perp}=\frac{2 R}{b^{2}} \frac{Z_{1}}{n \pm Q} .
$$

Table 2 shows the summary of data concerning the transverse resistive wall in the SPS and LHC. Its contents is commented in the following sections.

\subsection{Electrical properties of vacuum pipe}

Meaden [7] quotes $750 n \Omega m$ for the resistivity of stainless steel. In [8] I found $900 \mathrm{n} \Omega \mathrm{m}$. It is the last value that fits best the observed instability rise times in the SPS for standard high intensity beams in fixed target operation [9].

It is estimated that about $10 \%$ of the circumference of the LHC beam pipe will be at room temperature while the remaining $90 \%$ will be at 5 to $20^{\circ} \mathrm{K}$. To keep the contribution of the warm part within reasonable limits so that complicated systems as described in [10] are avoided, it has been proposed to use copper vacuum pipes where ever possible in the warm part $[11,12]$.

The cryogenic part of the LHC beam pipe (or rather beam screen) will be copper cladded stainless steel to keep resistance as low as possible both for considerations of instability and ohmic heating [13]. The resistivity of cold copper is a function of the 
residual resistance ratio $(R R R)$ and of the magnetic field $B$ [14]. The magnetic field increases the path length of the conduction electrons which leads to a substantial resistance increase at cryogenic temperatures. The final resistivity depends more on the field than on the $R R R$ for very high magnetic fields. The zero field $R R R=100$ is reduced to 80 at injection and to 30 in coast at $7 \mathrm{TeV} / \mathrm{c}$. It explains the existence of the Cu-low B and Cu-high B column in Table 2.

The skin depth $\delta$ can be larger than the thickness $d$ of the vacuum chamber wall for the lowest frequencies. The wall will not carry the full image current since a part of the magnetic field leaks out of the chamber wall. This effect reduces the effective resistance and it can be computed as an equivalent chamber thickness $d^{*}>d[15]$ :

$$
d^{*}=d\left(1+\left(\frac{\delta^{2}}{d b}\right)^{2}\right)
$$

It turns out that the outer stainless steel mantle in the LHC can be neglected here.

\subsection{Imaginary tune shift from resistive wall in partially filled machine}

The imaginary tune shift caused by the resistive wall is the convolution of the beam spectrum and the impedance characteristic. The beam of the fully charged LHC is nearly equally distributed around the circumference. The beam spectrum is more narrow than the impedance characteristic and the value of the beam current that enters the tune shift formula (1) is just the average or total intensity $i_{\text {tot }}$. In the SPS an interesting phenomenon occurs with the LHC type of beam. Indeed, only one quarter of the circumference is occupied by bunches when the machine is at maximum charge. The spectrum of the beam is not so narrow now and the convolution with the impedance also involves higher fast and slow wave modes so that some cancellation occurs as happens for the single bunch head tail effect. As a consequence the maximum effect of the impedance does not occur at the usual $n-Q=-0.4$ mode but at $n-Q=2.4$ where the convolution is largest. This is illustrated in Figure 3. 


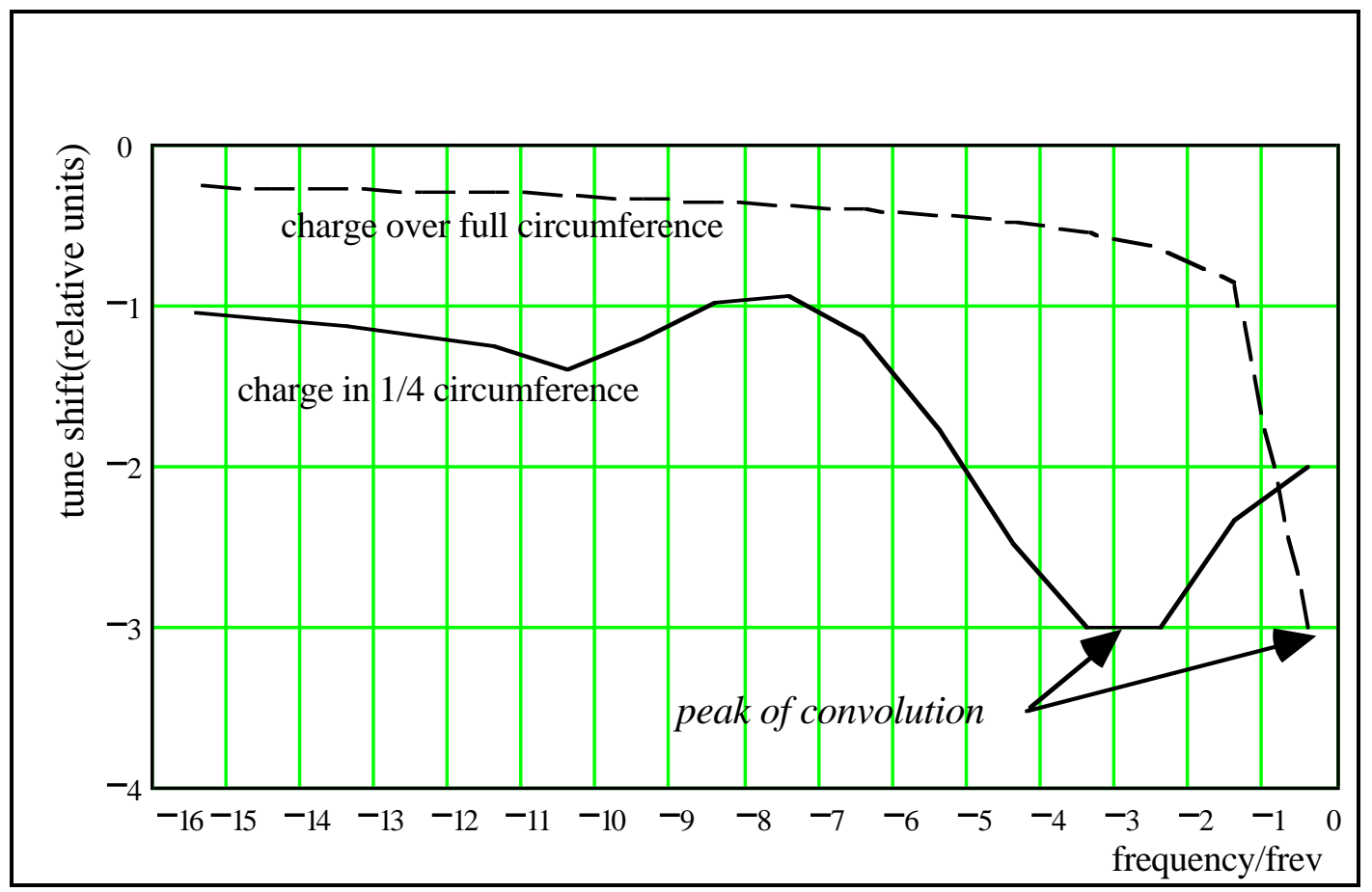

Figure 3 : Tune shift comparison between full and partially filled machine

It turns out that the magnitude of the imaginary tune shift is the same for an equally distributed charge or a charge concentrated in $1 / 4$ of the circumference.

\section{Real coherent tune shifts}

The computed imaginary tune shifts in Table 2 and their corresponding gains warrant clearly the need for a feedback system at the lower oscillation modes. The high frequency $\left(f_{b} / 2=20 \mathrm{MHz}\right)$ figures may lead to an erroneous conclusion. Indeed it may be tempting to say that tune spreads in the order of 0.0015 in the SPS and 0.00005 in the LHC are surely available from unavoidable non linearities. They certainly exist but they are utterly insufficient to provide the necessary Landau damping. This is due to the presence of real coherent tune shifts which must be taken into account when studying beam stability in a stability diagram [16].

Two possible sources ${ }^{2}$ of real coherent tune shift have to be considered. The first one is the reactive part of the transverse impedance. This impedance is inductive and defocusing with the exception of the horizontal plane in the SPS [17]. The second source is the single bunch coherent image tune shift [18]. There is no coherent tune shift relative to the total charge in the machine since the lowest oscillation frequency is still well shielded by the skin effect in the beam pipe [18].

The real tune shift caused by the inductive wall is computed with the standard formula (1). The inductive impedance is a very wide banded impedance and couples with the full bunch spectrum both in the case of the SPS and LHC where bunches are

\footnotetext{
2 the imaginary part of the wall skin effect impedance is neglected. It is smaller than the effects studied here even at the lowest modes where it reaches its maximum value.
} 
long with respect to transverse vacuum chamber dimensions. For that reason it is the peak intensity that has to be used in (1) in this particular case.

For the image tune shift the same peak intensity is used with the following formula :

$$
\Delta Q_{\text {image }}=-\frac{\hat{i}}{(2 \pi)^{2}} \frac{R / Q Z_{0} R}{(E / e) \gamma^{2}} \frac{\xi_{1}}{b^{2}}
$$

where $Z_{0}$ is the impedance in free space and $\xi_{1}$ the coherent tune shift factor. For the LHC the inscribed ellipse is used to define $\xi_{1}$. By combining the tune shift formulae for the transverse impedance (1) and for the image (6) a transverse image impedance can be defined which is inductive as well since both tune shifts have the same sign:

$$
Z_{\text {Limage }}=\frac{\xi_{1}}{\pi} \frac{Z_{0} R}{\gamma^{2} b^{2}} \cdot 3
$$

\begin{tabular}{|c|c|c|c|c|}
\hline & & SPS & \multicolumn{2}{|c|}{ LHC } \\
\hline & \multirow{7}{*}{$\begin{array}{l}\Omega \\
M \Omega / m \\
M \Omega / m \\
A\end{array}$} & & Cu-low B & Cu-high B \\
\hline (imaginary) & & 6 & \multirow{3}{*}{\multicolumn{2}{|c|}{$\begin{array}{c}0.25 \\
6.5 \\
0.004\end{array}$}} \\
\hline$Z_{\perp}$ & & $\leq 20$ & & \\
\hline$Z_{\text {Limage }}^{+}$ & & 0.16 & & \\
\hline \multirow{3}{*}{$\begin{array}{l}\text { peak current } \hat{i} \\
\Delta Q_{\perp} \quad(\text { real }) \\
\delta Q \text { spread for stability }\end{array}$} & & 10.9 & 25 & 42 \\
\hline & & 0.028 & 0.0019 & 0.0002 \\
\hline & & $\sim 0.1$ & $\sim 0.008$ & $\sim 0.0008$ \\
\hline
\end{tabular}

Table 3: Coherent tune shifts in SPS and LHC

It is clear that the coherent image tune shift is negligible but the formula reveals that smaller machines (smaller beam momentum) are more vulnerable to this effect.

The SPS inductive wall impedance value stems from actual measurements in the transverse plane. The corresponding longitudinal impedance deduced from formula (4) is much smaller than what is actually measured longitudinally. It proves that a major part of the longitudinal impedance does not contribute very much to the transverse one presumably due to the large chamber sizes that are involved (cavities and other transversely voluminous objects).

3 The conventional formula is $Z_{\text {Limage }}=\frac{\xi_{1}}{\pi} \frac{Z_{0} R}{\gamma^{2}}\left(\frac{1}{b^{2}}-\frac{1}{2 a^{2}}\right)$, where $a$ is the r.m.s. beam size. The inclusion of the space charge term $\left(1 / 2 a^{2}\right)$ in this formula is controversial. It does not vanish for increasing chamber radius $b$ as one would expect. Its effect is much larger than the chamber image term $\left(1 / b^{2}\right)$ and has the opposite sign. The computed space charge transverse impedance is very large in the SPS (-50 to $-75 \mathrm{M} \Omega / \mathrm{m})$ and would more than compensate the inductive wall $(<20 \mathrm{M} \Omega / \mathrm{m})$. However, the measured effect has the sign and magnitude of the inductive wall! One hypothesis is that the term is a computational residu arising from the fact that in the derivation of the formula the transverse beam edge has been considered as a fixed boundary while in reality it is a moving one. More theoretical work is needed to clarify this point. 
In the LHC the same kind of effort is being done as in LEP to keep the inductive impedance low. In LEP $Z / n \sim 0.25 \Omega$, excluding cavities. The same figure has been used in the table for the LHC.

From the last line in the table it becomes extremely clear that Landau damping at injection, even for the highest modes is not desirable. The tune spreads are too large for clean operation and the required strengths are far from being trivial. It may be interesting to compute the necessary integrated octupole field to stabilise the beam $[9,16]$.

The tune spread at $1 \sigma$ produced by octupoles is : $\delta Q_{4}=\frac{3}{16 \pi} \beta^{2} \frac{\varepsilon}{\gamma} S_{4}$.

The octupole field is defined as $(N=4)$ :

$$
S_{N}=\int \frac{b_{N} d l}{B \rho}=\frac{1}{(N-1) !} \int \frac{B^{(N-1)} d l}{B \rho} \quad \text { and } \quad B=b_{N} x^{N-1} .
$$

This yields following integrated octupole fields:

\begin{tabular}{|l|c|c|c|}
\hline & SPS & LHC low B & LHC high B \\
\hline $\mathrm{S}_{4}$ at average $\beta$ & $725 \mathrm{~m}^{-3}$ & $260 \mathrm{~m}^{-3}$ & $430 \mathrm{~m}^{-3}$ \\
$\mathrm{~S}_{4}$ at maximum $\beta$ & $125 \mathrm{~m}^{-3}$ & $40 \mathrm{~m}^{-3}$ & $65 \mathrm{~m}^{-3}$ \\
\hline
\end{tabular}

Table 4 : Octupole field to stabilise LHC beam.

\section{Damping of injection oscillations}

Injection errors blow up the emittance of a beam that is transversely stable (feedback) [19]. The emittance increase is caused by the decoherence [20] of the injection oscillation which depends upon the tune spread $\delta Q$ in the beam. The emittance increment with active feedback depends on the combination of injection error amplitude, tune spread and extra damping (above the needs for stability) by the feedback [21]. It can be shown that the emittance increment $\Delta \varepsilon$ can be written as [22]:

$$
\Delta \varepsilon=\frac{1}{2} e_{i n j}^{2}\left(\frac{1}{1+\Delta Q_{i n j} / \delta Q}\right)^{2}
$$

where $\Delta Q_{i n j}$ is the net tune shift imposed by the feedback system on injection errors $e_{i n j}$. In order to control the emittance increment, injection errors and tune spread have to be evaluated.

\subsection{Injection errors}




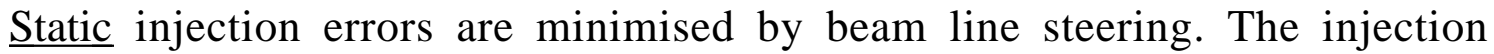
dampers take care of the time varying part of the injection errors: fast errors on the injection kicker pulse, slow variations due to power supply ripple of beam line bending magnets, including septa will cause differences between successive injections. The fast kicker rise times define the bandwidth where the full corrective power of the feedback with respect to these errors is required.

\section{- fast kickers}

The strength of fast kickers is essentially determined by the jump that the beam makes at the septum. By examining the extraction of the CPS and SPS and the injection of the SPS and the LHC it is interesting to note that the fast kicker deflection normalised for $\beta$ and $\gamma$ is the same within $10 \%$ :

$$
\Delta x \sqrt{\frac{\gamma}{\beta}}=0.1 \sqrt{m} .
$$

The kicker error can be estimated at $510^{-3}$ [23]. Combining 2 kickers yields :

$$
e_{K}=0.7 \sqrt{\mu} \leq 0.5 \sigma
$$

The error is mainly horizontal, but local tilts will make it also appear in the vertical plane ${ }^{4}$.

The fast kickers upstream of the SPS damper have a rise time shorter than 100 ns(CPS) which requires a (power) bandwidth of $5 \mathrm{MHz}$. The rise time of the fast kickers upstream of the LHC dampers is $1 \mu$ s requiring a (power) bandwidth of 0.5 $\underline{\mathrm{MHz}}$

\section{- bending magnets and septa ripple in transfer line}

The global ripple on the bending magnets in the transfer line between PS and SPS yields about the same error, but in both planes this time :

$$
e_{B}=0.5 \sigma
$$

By lack of further data the same value is assumed for the LHC injection lines. Septa, extraction and injection combined, yield about half the previous value.

$$
e_{S}=0.25 \sigma
$$

This error is mainly horizontal but coupling will again cause some leakage to the vertical plane as for the fast kickers.

\footnotetext{
${ }^{4}$ At the time of writing it was brought to my attention that the injection in the LHC will be done in the vertical plane. This will increase the $e_{K}^{2}$ and will reduce the major part of the rounding margin of (15).
} 
The total expected vertical injection error can be estimated by combining (12), (13) and (14):

$$
e_{i n j}^{2}=\frac{\gamma}{\beta}\left(e_{K}^{2}+e_{B}^{2}+e_{S}^{2}\right)=0.375 \frac{\gamma \sigma^{2}}{\beta} \Rightarrow \frac{1}{2} \varepsilon,
$$

where it is recalled that $\varepsilon$ is the unperturbed normal emittance of the injected beam. The result is slightly more optimistic than the assumptions made for the late SSC [21]. They are in fair agreement with the operational experience of the $S p \bar{p} S$.

\subsection{Tune spreads in the beam}

Tune spreads may arise from incoherent tune shifts. Non-linearities in the magnets will not be considered here since every effort will be made to keep them as small as possible in the LHC and they are very small in the SPS. Other possible sources of tune spread are the direct and image incoherent tune shifts [18,24]. Three cases are considered.

First the direct space charge, computed with :

$$
\Delta Q_{s c}=-\frac{\varepsilon_{s c} / 2}{\sigma^{2}} \frac{\hat{i}}{2 \pi} \frac{R / Q Z_{0} R}{(E / e) \gamma^{2}} \quad\left[\varepsilon=\frac{\gamma}{R / Q} \sigma^{2}\right] .
$$

Next the single bunch incoherent image tune shift :

$$
\Delta Q_{\text {image sb }}=-\frac{\varepsilon_{1}}{b^{2}} \frac{\hat{i}}{2 \pi} \frac{R / Q Z_{0} R}{(E / e) \gamma^{2}}
$$

Finally the incoherent tune shift created by the total charge in the machine :

$$
\Delta Q_{\text {image tot }}=-\frac{\varepsilon_{1}}{b^{2}} \frac{i_{\text {tot }}(R / Q) Z_{0} R}{2 \pi(E / e)} .
$$

The LHC chamber was approximated with the inscribed ellipse as for the coherent tune shifts before. The single bunch tune shift varies along the bunch intensity profile which causes a tune variation which can be estimated at roughly $3 / 4$ of the peak tune shift. The variation of the total intensity tune shift comes from the variation of the image factor $\varepsilon_{l}$ over the beam radius $\sigma$. This variation is at most a few percent only and can therefore be neglected. Table 5 summarises the data on tune shifts $(\Delta Q)$ and corresponding tune spreads $(\delta Q)$.

\begin{tabular}{|l|c|c|c|c|}
\hline & & SPS & LHC low B & LHC high B \\
\hline peak intensity & $A$ & 10.9 & 25 & 42 \\
average intensity & $A$ & 0.29 & 0.87 & 0.87 \\
$\varepsilon$ & $\mu \mathrm{radm}$ & 3 & 3.5 & 3.75 \\
$\Delta Q_{s c}$ & & 0.067 & 0.0017 & 0.00001 \\
$\delta Q_{s c}$ & & $\mathbf{0 . 0 5}$ & $\mathbf{0 . 0 0 1 3}$ & $\mathbf{0 . 0 0 0 0 0 8}$ \\
$\Delta Q_{\text {image } s b}$ & & 0.0006 & 0.00001 & -
\end{tabular}




\begin{tabular}{|l|c|c|c|c|}
$\delta Q_{\text {image sb }}$ & 0.0004 & 0.000008 & - \\
$\Delta Q_{\text {image tot }}$ & 0.01 & 0.01 & 0.0006 \\
$\delta Q_{\text {image tot }}$ & - & - & - \\
\hline
\end{tabular}

Table 5: Tune spreads in SPS and LHC

The direct effect is dominant but still rather insufficient for Landau damping as can be seen from the data presented in Table 3.

\subsection{Damping of injection errors}

Formula (10) computes the necessary feedback tune shift, hence feedback gain, for any given emittance blow-up budget $\Delta \varepsilon$. This gain has to be added to the gain that is needed to stabilise the beam. The total gain and the maximum expected injection error $e_{i n j}$ yields the maximum deflection $\theta$ that is required :

$$
\theta=G_{t o t a l} \sqrt{\frac{\varepsilon / 2}{\beta_{k i c k e r} \gamma}}
$$

The deflection $\theta$ is delivered by a deflector with an integrated transverse electric or magnetic field or both (stripline kicker, strength reduced by factor 2) of the following magnitude :

$$
c \int B_{\perp} d l=\int E_{\perp} d l=G_{\text {total }} \sqrt{\frac{\gamma \varepsilon / 2}{\beta_{k i c \mathrm{ker}}}} \frac{E_{0}}{e} .
$$

Table 6 shows the strength of the kickers if they were located at a place where the optical parameter $\beta$ is maximum. Obviously, this strength increases when $\beta$ is less than maximum.

\begin{tabular}{|l|l|c|c|}
\hline & & SPS & LHC \\
\hline allowed blow-up & $\mu r a d m$ & 0.4 & 0.2 \\
$e_{\text {inj }}^{2}$ & $\mu r a d m$ & 1.5 & 1.75 \\
tune spread $\delta Q$ & & 0.05 & 0.0013 \\
required tune shift $\Delta Q_{\text {inj }}$ & & 0.019 & 0.0014 \\
gain & & 0.23 & 0.018 \\
total gain & & 0.313 & 0.0264 \\
deflection (max of $\beta$ ) & $\mu r a d$ & 7.2 & 0.12 \\
$\int E_{\perp}$ dl & $k V$ & 190 & 56 \\
$\int B_{\perp}$ dl & $\mu T m$ & 620 & 190 \\
power band-width & $M H z$ & 5 & 0.5 \\
\hline
\end{tabular}

Table 6 : Gain and deflection requirements for SPS and LHC feedback

\section{Emittance conservation during injection coast and acceleration}


It is obvious that the feedback must stay active outside the injection times to keep the beam stable. The beam, driven by the instability, will oscillate during that time up to the observation limit of the system. The observation limit is determined by the highest noise level in the feedback loop. If digital processes are used then this level can be $\mathrm{LSB} / \sqrt{ } 12$ if it is larger than the equivalent analog noise. The persistent oscillation will blow up the beam emittance according to [22]:

$$
\frac{d \varepsilon}{d t}=\frac{\gamma}{\beta} \frac{\bar{x}^{2}}{T} \delta Q
$$

where $\bar{x}$ is the r.m.s. noise level expressed in units of beam position. Most position monitors are sensitive to the dipole moment of the beam. Hence the position resolution will be worst with the lowest intensity. Table 7 gives a summary assuming a noise figure of $20 \mathrm{~dB}$ in the BPM processing electronics, which is a reasonable value for several types of processing.

\begin{tabular}{|l|l|c|c|}
\hline & & SPS & LHC(accel) \\
\hline noise level & $\mu V$ & 40 & 40 \\
effective monitor $Z$ & $\Omega / m$ & 40 & 1605 \\
resolution & $A \bullet \mu$ & 1 & 0.25 \\
average coast time & $s e c$ & 7.2 & $200(1200)$ \\
max blow-up & $\mu \mathrm{radm}$ & 0.1 & 0.05 \\
max rate & $\mu \mathrm{radm} / \mathrm{se}$ & 0.014 & 0.00004 \\
& $c$ & & \\
max $\bar{x}$ for max rate & $\mu$ & $\mathbf{5}$ & $\mathbf{1}$ \\
dynamic & & 16000 & 20000 \\
reduced dynamic & & $\mathbf{2 2 0 0}$ & $\mathbf{3 8 0 0}$ \\
analog $\bar{x}$ for ultimate $(1.09 \mathrm{~A})$ & $\mu$ & 0.9 & 0.23 \\
analog $\bar{x}$ for nominal $(0.64 \mathrm{~A})$ & $\mu$ & 1.5 & 0.4 \\
\hline
\end{tabular}

Table 7 : Resolution and emittance blow-up in SPS and LHC

The analog resolution is adequate. The required dynamic range is impressive. If digital processing is included then the dynamic range has to be reduced but such that the resolution is maintained. This can be done by reducing the electronic acceptance to a small part of the total aperture (electronic acceptance zoom). This (electronic) aperture must accommodate the injection errors $(1.4 \sigma)$ and the orbit $(2 \sigma)$ giving a total of $3.4 \sigma$. The computed numbers are compatible with a 10 bit digitisation and this is an encouraging fact.

This phase of the feedback operation follows immediately the high power injection phase. During the high power phase very large signals are handled, while the system is operating just above the noise level during the low power phase. The deflection power is needed up to the power band-width. It can be envisaged for example, that the low frequency signals are directed towards the driver of a high deflection kicker and the high frequency signals to the driver of a low deflection kicker. The difference in required deflection between them is large (90 dB SPS and

5 cable length of $200 \mathrm{~m}$ taken into account for processing frequency of $80 \mathrm{MHz}$. 
$105 \mathrm{~dB}$ LHC) . It is composed on one hand by the ratio of the maximum injection error and the BPM resolution (55 dB SPS and $60 \mathrm{~dB}$ LHC) and on the other hand by the gain ratio between the lowest and highest feedback frequency (35 dB SPS and $45 \mathrm{~dB}$ LHC). The data in Table 8 summarises some of the relevant data in this respect.

\begin{tabular}{|l|l|c|c|}
\hline \multicolumn{2}{|c|}{} & SPS & LHC \\
\hline damping time injection errors & turns & 9 & 140 \\
HF limit injection errors & $\mathrm{MHz}$ & 5 & 0.5 \\
$\int E_{\perp}$ dl needed for injection errors & $\mathrm{kV}$ & 190 & 56 \\
growth rate instability at HF limit & turns & 200 & 2000 \\
growth rate instability at $20 \mathrm{MHz}$ & turns & 400 & 13000 \\
$\int E_{\perp}$ dl needed at HF limit inj. errors & $\mathrm{V}$ & 13 & 2.5 \\
$\int E_{\perp}$ dl needed at $20 \mathrm{MHz}$ & $\mathrm{V}$ & 6.5 & 0.46 \\
\hline
\end{tabular}

Table 8 : Strength of high power and high bandwidth feedback

\section{Emittance conservation during long collider coasts $[25,26]$}

Only the LHC is concerned. The tune spread in collision is expected to be 0.015 [27]. This switches off the instability. If the feedback remains active its noise will cause a blow-up with following rate:

$$
\frac{d \varepsilon}{d t}=\frac{1}{2} \frac{\gamma}{\beta} \frac{\bar{x}^{2}}{T} G^{2} .
$$

If the feedback is left in the same state as the one required during the acceleration phase then loop noise would blow up the beam emittance with a rate of 3 hours, which is unacceptable. It has been shown that ground motion can cause occasionally growth times of less than 40 hours. Other low frequency noise sources certainly exist (corrector power supplies). A small but beneficial effect comes from synchrolight damping which tends to reduce the transverse emittance at a rate of $\sim 60$ hours. In any case, a coast feedback which limits the persistent oscillation amplitude to $50 \mathrm{~nm}$ can take care of the noises and at the same time it will provide extra operational security. With digital techniques it is almost trivial to reduce the bandwidth from $20 \mathrm{MHz}$ to $f_{r e v}=11 \mathrm{kHz}$ in a digital signal processing unit. The noise level will reduce by a factor 40 ( $1 \mu$ to $25 \mathrm{~nm}$ ). The gain of the feedback needs to be increased but not too much else the cure is worse than the remedy. Increasing the gain to $G>4 \pi \Delta Q=0.19$ will cause a very acceptable noise lifetime of 180 hours. The potential emittance increase

\footnotetext{
${ }^{6}$ It turns out that at $7 \mathrm{TeV} / \mathrm{c}$ about $10 \%$ more integrated field is necessary.
} 
of noise sources reduces by $3 / 4$ and ground motion noise effects would be reduced by the same factor so that its emittance growth rate increases to 160 hours.

\section{Conclusions}

Several aspects of beam behaviour have been studied to check their implications on a transverse feedback system for the LHC type beam in the SPS and LHC. The system that emerges from this investigation is feasible in terms of analog circuits elements: position monitors and processing, deflectors and associated drivers, operating frequency and band width. However, the operational phases are extremely varied and complex such that the use of Digital Signal Processing techniques will not be a luxury but a necessity.

\section{References}

[1] E. Keil, W. Schnell and P. Strolin, Feedback Damping of Horizontal Beam Transfer Errors, CERN 69-27, 1969.

[2] G. Rochepeau, e.a., Damping of Transverse Oscillations in the ISR, VIIIth Conference on High Energy Accelerators, CERN, 1971.

[3] J. Crisp, e.a., A programmable High Power Beam Damper for the Tevatron, IEEE, NS-32, 1985.

[4] D. Heins, e.a., Wide Band Multi-Bunch Feedback Systems for Petra, DESY 89$157,1989$.

[5] R. Bossart, e.a., Operation of the Transverse Feedback System at the CERN SPS, PAC 1987.

[6] L. Vos, Transverse Feedback System in the CERN SPS, CERN SL/91-40 (BI), 1991.

[7] G. T. Meaden, Electrical Resistance of Metals, London, 1966

[8] Reference Data for Radio Engineers, ITT, Howard W. Sams \&Co.,Inc. 1975.

[9] L. Vos, Transverse Stability Considerations for the SPS Beam in Fixed Target Operation, CERN SPS/86-3 (DI-MST), 1986.

[10] I.N. Ivanov, e.a. Requirements to the Feedback System to damp resistive instability (I stage UNK) XVth ICHEA, Hamburg, 1992

[11] P. Lefèvre, editor, The Large Hadron Collider, CERN/AC/95-05(LHC), 1995

[12] F. Ruggiero, Single-Beam Collective Effects in the LHC, CERN SL/95-09 (AP), 1995.

[13] L.Vos, Beam Vacuum Chamber Effects in the Large Hadron Collider, CERNSPS/84-10 (DI-MST) 1984

[14] M. G. Benz, The magnet resistance of copper at $4.2^{\circ} \mathrm{K}$ in transverse fields up to 100 kilogauss, General Electric Report No. 68-C-416, 1968.

[15] B. Zotter, Transverse Oscillations of a Relativistic Particle Beam in a Laminated Vacuum Chamber, CERN 69-15, 1969.

[16] L. Vos, Transverse Stability of the High Intensity SPS Proton Beam, EPAC, Rome, 1988.

[17] L. Vos, Computer Calculation of the Longitudinal Impedance of Cylindrically Symmetric Structures and its Application to the SPS,PAC 1987. 
[18] L. J. Laslett, On Intensity Limitations imposed by Transverse Space-charge Effects in Circular Particle Accelerators, Proc. Brookhaven Summer Study, BNL $7534,1963$.

[19] H.Hereward, How good is the R.M.S. as a measure of Beam Size? CERN/MPS/DL 69-15, 1969.

[20] K. Hübner, CERN Report ISR-TH/69-17, 1969.

[21] G. R. Lambertson, Feedback to suppress Beam Instabilities in Future Proton Rings, IEEE, Vol. NS-32, 1985.

[22] L. Vos, Report in preparation.

[23] Ducimetière L., e.a., Design of the Injection Kicker Magnet System for CERN's 14 TeV Proton Collider LHC, CERN-SL-95-80 BT, 1995.

[24] G. Guignard, Selection of Formulae concerning Proton Storage Rings, CERN 77$10,1977$.

[25] V. A. Lebedev, The Super Collider Transverse Feedback System for Suppression of the Emittance Growth and Beam Instabilities, Budkerinp 93-61, 1993.

[26] L. Vos, Ground Motion in LEP and LHC, CERN SL/94-69 (BI), 1994.

[27] W. Herr, Beam-beam effects in the LHC, Particle Accelerators 50, 1994.

[28] W. Barry, G. R. Lambertson and C. C. Lo, Electronic Systems for Transverse Coupled-bunch Feedback in the Advanced Light Source, LBL-34405, 1993. 